\title{
Early Infliximab Yields Superior Long-Term Effects on Linear Growth in Pediatric Crohn's Disease Patients
}

\author{
Jaeyoung Choi ${ }^{1}$, Ben Kang ${ }^{2,3}$, Min-Ji Kim ${ }^{4}$ Insuk Sohn ${ }^{4}$, Hae Jeong Lee ${ }^{5}$, and Yon Ho Choe ${ }^{1}$ \\ ${ }^{1}$ Department of Pediatrics, Samsung Medical Center, Sungkyunkwan University School of Medicine, Seoul, ${ }^{2}$ Department of Pediatrics, School \\ of Medicine, Kyungpook National University, ${ }^{3}$ Crohn's and Colitis Association in Daegu-Gyeongbuk (CCAiD), Daegu, ${ }^{4}$ Biostatistics and Clinical \\ Epidemiology Center, Samsung Medical Center, Seoul, and ${ }^{5}$ Department of Pediatrics, Samsung Changwon Hospital, Sungkyunkwan University \\ School of Medicine, Changwon, Korea
}

\section{See editorial on page 221}

Background/Aims: Information regarding the efficacy of early infliximab treatment in pediatric patients with Crohn's disease (CD) is limited. We aimed to evaluate the impact of early combined immunosuppression on linear growth in pediatric patients with CD by performing step-up comparisons. Methods: This retrospective study included pediatric patients with moderate-to-severe $\mathrm{CD}$, who received a combination therapy with infliximab and azathioprine for at least 3 years and sustained corticosteroid-free remission without loss of response. The z-scores of the growth indicators obtained at the time of diagnosis and annually for 3 years thereafter were compared between the two groups. Results: The early combined immunosuppression group displayed significantly increased linear growth 3 years after diagnosis $(p=0.026)$. A significant difference was also observed in the linear growth 3 years after diagnosis between subgroups of Tanner stages 1-2 ( $p=0.016)$. Conclusions: The early introduction of biologics should be considered to improve linear growth in pediatric patients with CD. (Gut Liver 2018;12:255-262)

Key Words: Infliximab; Growth; Height; Tanner stage

\section{INTRODUCTION}

The conventional approach for treatment of pediatric Crohn's disease (CD) before the recent consensus guidelines of European Crohn's and Colitis Organisation (ECCO) and European Society for Paediatric Gastroenterology Hepatology and Nutrition (ESP-
GHAN) ${ }^{1}$ had been constituted of inducing remission with corticosteroid (CS) and maintaining remission with immunomodulators. ${ }^{2}$ Infliximab (IFX) had been considered for those refractory or intolerant to conventional treatment, known as the step-up approach. ${ }^{3,4}$ On the other hand, several clinical trials have introduced the concept of early combined immunosuppression, supporting the early use of IFX and immunomodulators before the use of CS for CD patients with certain high risks.,

Data comparing the efficacy of the two approaches on growth of children are limited. In our previous study, no significant differences were found in z-scores for height, weight, and body mass index (BMI) between the two strategies at 3 years after IFX initiation. ${ }^{7}$ In this study, however, patients that had stopped IFX after the first year were also included, resulting in a decline of z-scores for height 3 years after IFX treatment, which is contrary to previous studies. ${ }^{3,8-12}$ Furthermore, the extension to the REACH study (a randomized, multicenter, open-label study to evaluate the safety and efficacy of anti-TNF $\alpha$ chimeric monoclonal antibody in pediatric subjects with moderate-tosevere Crohn's disease) ${ }^{13}$ and recent study by Cameron et al. ${ }^{14}$ showed better growth improvement in patients under remission, suggesting difference in growth restoration depending on disease control status. Therefore, in order to investigate the longterm effect of combined immunosuppression on linear growth with exclusion of the influence of disease status, we aimed to investigate the impact of early combined immunosuppression on linear growth in pediatric CD patients who had continuously received combined immunosuppression with IFX and azathioprine (AZA) and had sustained remission for 3 years, by comparison with those who had stepped-up to receive combined immunosuppression and had sustained remission for 3 years.

\footnotetext{
Correspondence to: Yon Ho Choe

Department of Pediatrics, Samsung Medical Center, Sungkyunkwan University School of Medicine, 81 Irwon-ro, Gangnam-gu, Seoul 06351, Korea Tel: +82-2-3410-3527, Fax: +82-2-3410-0043, E-mail: yonho.choe@samsung.com

Received on July 4, 2017. Revised on September 11, 2017. Accepted on September 22, 2017. Published online January 5, 2018 pISSN 1976-2283 eISSN 2005-1212 https://doi.org/10.5009/gnl17290 Jaeyoung Choi and Ben Kang contributed equally to this work as first authors.

(c) This is an Open Access article distributed under the terms of the Creative Commons Attribution Non-Commercial License (http://creativecommons.org/licenses/by-nc/4.0) which permits unrestricted non-commercial use, distribution, and reproduction in any medium, provided the original work is properly cited.
} 
In addition, we aimed to compare the effect of IFX on linear growth between subgroups of patients further divided according to Tanner stages.

\section{MATERIALS AND METHODS}

\section{Patient enrollment}

This study was a retrospective observational cohort conducted at the Department of Pediatrics of Samsung Medical Center from January 2008 to December 2016. Enrolled patients were those diagnosed with moderate to severe luminal CD with nonpenetrating and nonstricturing behavior who had started IFX and AZA before the age of 18. Among these patients, those who had continuously received combined immunosuppression for at least 3 years and had sustained CS-free remission without loss of response (LOR) were included in this study, in order to minimize the effect of unregulated inflammation of $\mathrm{CD}$ on growth.

Patients who had stopped IFX before 3 years of treatment, or those who had received dose intensification of IFX due to LOR were excluded. Patients with previous history of bowel surgery, or IFX indicated for the treatment of refractory perianal fistulas were excluded. The diagnosis of $\mathrm{CD}$ was made in accordance with the ESPGHAN-Porto criteria. ${ }^{15}$ Moderate to severe luminal $\mathrm{CD}$ was defined as a score of 30 points or more on the Pediatric Crohn's Disease Activity Index (PCDAI). ${ }^{16}$ Disease classification and behaviour was based on the Paris classification. ${ }^{17}$

\section{Study design}

Since 2008 we have employed a treatment protocol in which patients and guardians were allowed to choose their initial treatment between either a conventional step-up strategy or an early combined immunosuppression strategy in children newly diagnosed with moderate-to-severe $\mathrm{CD}$. After thorough explanation of the pros and cons of each treatment strategy, patients and guardians were given the choice to whether step-up treatment or initiate treatment IFX within 1 month from diagnosis. The investigators did not involve in the decision of which treatment strategy would be initiated.

Table 1. Comparison of Baseline Characteristics in the Two Groups

\begin{tabular}{|c|c|c|c|}
\hline Characteristic & Step-up $(\mathrm{n}=16)$ & Early combined immunosuppression $(\mathrm{n}=17)$ & p-value \\
\hline Male sex & $11(69)$ & $11(64)$ & 0.806 \\
\hline Tanner stage $1-2$ at diagnosis & $10(63)$ & $8(47)$ & 0.373 \\
\hline Tanner stage $1-2$ at IFX infusion & $8(50)$ & $8(47)$ & 0.866 \\
\hline Lower GI location & $16(100)$ & $17(100)$ & 0.498 \\
\hline L1 & $1(6)$ & $2(12)$ & \\
\hline $\mathrm{L} 2$ & 0 & $2(12)$ & \\
\hline L3 & $15(94)$ & $13(76)$ & \\
\hline Upper GI location & $16(100)$ & $17(100)$ & 0.208 \\
\hline No involvement & $7(43)$ & $10(59)$ & \\
\hline L4a & $2(13)$ & $2(12)$ & \\
\hline $\mathrm{L} 4 \mathrm{~b}$ & $3(19)$ & $5(29)$ & \\
\hline $\mathrm{L} 4 \mathrm{a}+\mathrm{b}$ & $4(25)$ & 0 & \\
\hline Perianal fistula & $12(75)$ & $9(53)$ & 0.188 \\
\hline Age at diagnosis, yr & $12.1(9.1-15.6)$ & $15.0(9.9-16.7)$ & 0.009 \\
\hline Age at IFX initiation, yr & $14.2(10.4-16.9)$ & $15.1(10.0-16.8)$ & 0.407 \\
\hline PCDAI & $35.0(30.0-60.0)$ & $40.0(30.0-60.0)$ & 0.908 \\
\hline $\mathrm{WBC}, / \mu \mathrm{L}$ & $8,455(4,750-16,220)$ & $8,320(3,970-13,210)$ & 0.643 \\
\hline Hematocrit, \% & $33.8(27.5-39.2)$ & $33.0(26.0-40.0)$ & 0.928 \\
\hline Platelet count, $\times 10^{3} / \mu \mathrm{L}$ & $424(330-672)$ & $378(287-680)$ & 0.796 \\
\hline ESR, $\mathrm{mm} / \mathrm{hr}$ & $72.5(45.0-120.0)$ & $69.0(28.0-99.0)$ & 0.349 \\
\hline CRP, mg/dL & $2.2(0.5-6.2)$ & $2.7(0.7-7.5)$ & 0.159 \\
\hline Albumin, $\mathrm{g} / \mathrm{dL}$ & $3.6(2.9-4.5)$ & $3.6(2.3-4.3)$ & 0.803 \\
\hline Duration from diagnosis to IFX initiation, mo & $11.4(1.5-68.5)$ & $0.7(0.1-1.0)$ & $<0.001$ \\
\hline
\end{tabular}

Data are presented as number (\%) or median (range).

IFX, infliximab; GI, gastrointestinal; L1, distal 1/3 ileum \pm limited cecal disease; L2, colonic disease; L3, ileocolonic disease; L4a, upper disease proximal to ligament of Treitz; L4b, upper disease distal to the ligament of Treitz and proximal to the distal 1/3 ileum; L4a+b, upper disease involvement in both L4a and L4b; PCDAI, Pediatric Crohn's Disease Activity Index; WBC, white blood cell; ESR, erythrocyte sedimentation rate; $\mathrm{CRP}, \mathrm{C}$-reactive protein. 
Patients in the step-up group received oral CS of $1 \mathrm{mg} / \mathrm{kg} /$ day (maximum $60 \mathrm{mg} /$ day) for induction therapy which was tapered over 8 weeks, and oral AZA and mesalazine were provided for maintenance therapy. When the patients in the step-up group proved to be refractory to conventional treatment or dependent to CS, IFX was administered without changes in AZA and mesalazine. IFX was also indicated when clinical relapse was observed during maintenance of clinical remission with AZA and mesalazine. Clinical remission was defined as a PCDAI of 10 points or under, and clinical relapse was defined as a PCDAI of more than 10 points after achieving clinical remission. $\mathrm{Pa}-$ tients in the early combined immunosuppression group received intravenous IFX along with oral AZA and mesalazine within 1 month from diagnosis.

In both groups, IFX had been administered with a dosage of

A

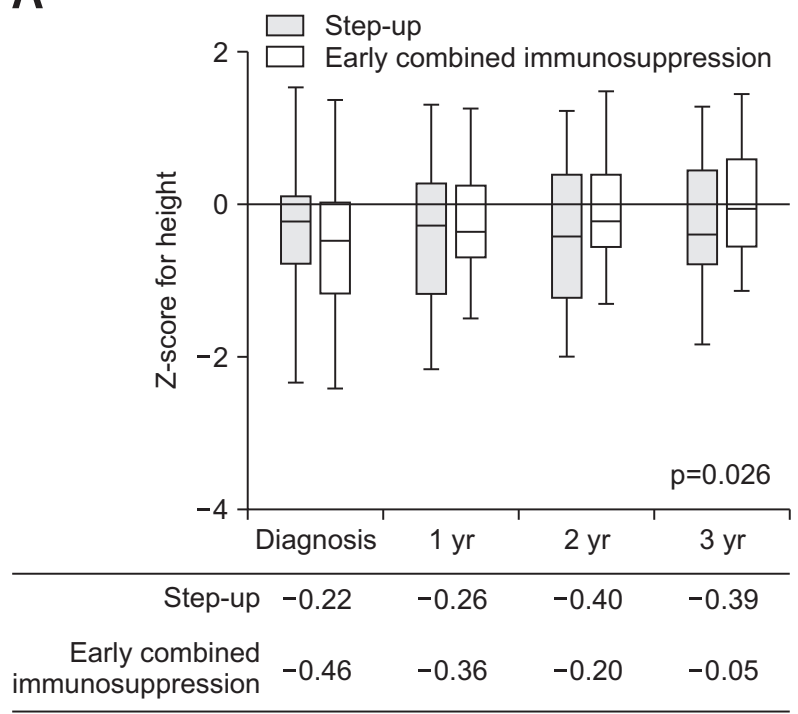

C

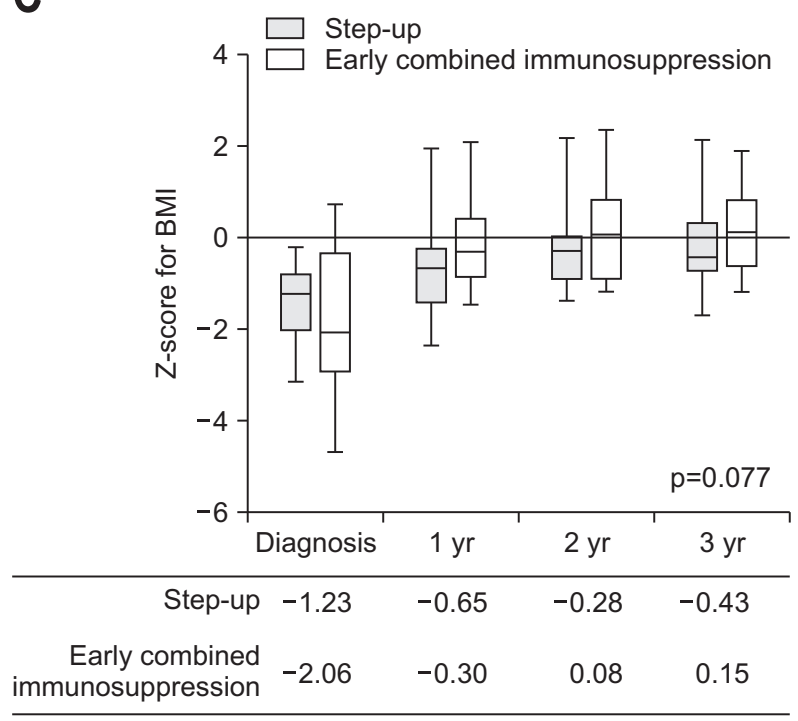

$5 \mathrm{mg} / \mathrm{kg}$ by scheduled infusion at weeks 0,2 , and 6 during the induction period, and was repeated every 8 weeks thereafter. AZA was initiated at doses of 0.5 to $1 \mathrm{mg} / \mathrm{kg} /$ day. The dose of AZA had been modified after initiation when required based on adverse events, laboratory exams, thiopurine methyltransferase genotype results, and thiopurine metabolite levels of 6-thioguanine nucleotides and 6-methylmercaptopurine. ${ }^{18,19}$ Patients in both groups had received partial enteral nutrition (EN) support during the IFX induction phase.

Baseline clinicodemographic characteristics at diagnosis, 1 year, 2 years, and 3 years after diagnosis were obtained in the two groups. Tanner stage at diagnosis, sex, birth date, disease location, and presence of anal fistula and other clinicodemographic data, including PCDAI scores were also obtained. Serum laboratory exams of complete blood cell counts with differential

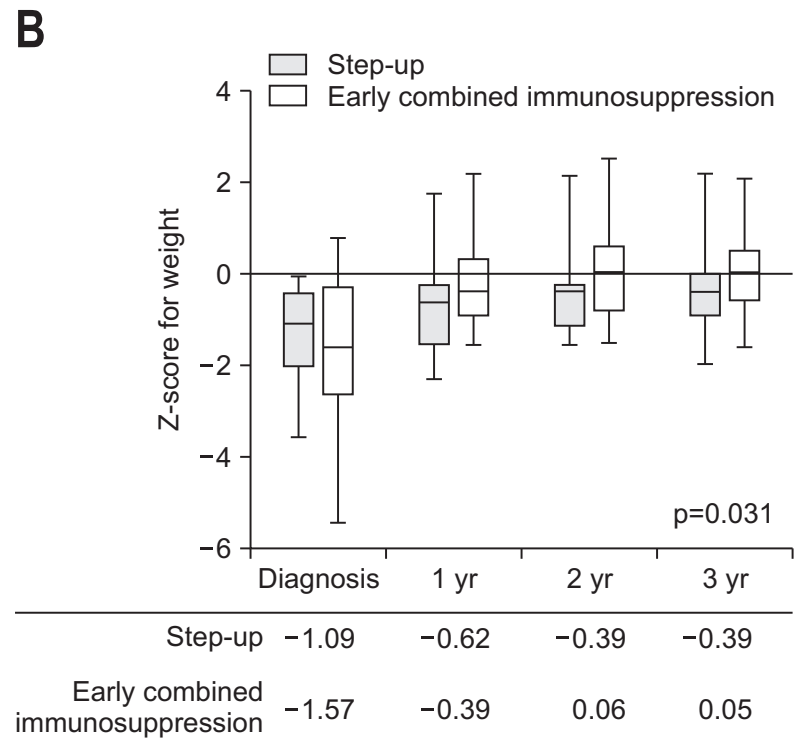

Fig. 1. Box-and-whisker plots of the $z$-scores of the growth indicators since the time of diagnosis. Box-and-whisker plot of the zscores of height, weight and body mass index (BMI) showing the median (line in box); 25th and 75th percentiles (box ends) and minimum and maximum (whiskers) values. Height z-scores each year after diagnosis (A); weight z-scores each year after diagnosis (B); and BMI z-scores each year after diagnosis (C). The p-values represent an interaction effect between the treatment strategies and time in the generalized estimating equation analysis. The table below represents the median z-scores of height, weight, and BMI in each group shown in (A), (B), and (C), respectively. 
counts, chemistry profiles, erythrocyte sedimentation rate, Creactive protein were performed on each outpatient visit. PCDAI scores were calculated at each visit to assess the efficacy of treatment. z-scores for height, weight, and BMI were calculated based on the growth charts of the Korea Centers for Disease Control and Prevention. ${ }^{20} \mathrm{z}$-scores for these growth indicators were compared between the two groups. Analyses between subgroups of patients further divided according to Tanner stages were also conducted. Tanner stages were divided into lower Tanner stage of 1-2 and higher Tanner stage of 4-5 as done in a previous study. ${ }^{12}$

\section{Statistical methods}

Wilcoxon two-sample test and paired t-test were used for the comparison of continuous variables, based on the results of
Shapiro-Wilk test for normality. Fisher exact test and chi-square test were used for the comparison of categorical variables. Generalized estimating equations (GEE) analysis with unstructured correlation was used to evaluate the time effect and the interaction effect between the two treatment strategies and time on the z-scores for growth indicators. Post-hoc comparison and Bonferroni correction were used for multiple comparisons. The $p$-value for statistical significance was defined as $p<0.05$. Statistical analysis was carried out on the SAS version 9.3 (SAS Institute, Cary, NC, USA).

\section{Ethics statement}

This study was approved by the Institutional Review Board of Samsung Medical Center (IRB number: 2015-01-047-002) and was conducted in accordance with the Declaration of Helsinki.

Table 2. GEE Analysis of the $\mathrm{z}$-Scores of the Growth Indicators 3 Years after Diagnosis $(n=33)$

\begin{tabular}{|c|c|c|c|c|c|c|c|c|c|}
\hline \multirow{2}{*}{ Factor } & \multicolumn{3}{|c|}{ Height } & \multicolumn{3}{|c|}{ Weight } & \multicolumn{3}{|c|}{ BMI } \\
\hline & $\chi^{2}$ & $\mathrm{DF}$ & $\mathrm{p}$-value & $\chi^{2}$ & $\mathrm{DF}$ & p-value & $\chi^{2}$ & $\mathrm{DF}$ & p-value \\
\hline Intercept & 0.069 & 1 & 0.793 & 0.068 & 1 & 0.794 & 0.015 & 1 & 0.904 \\
\hline Sex & 0.260 & 1 & 0.610 & 0.69 & 1 & 0.406 & 1.455 & 1 & 0.228 \\
\hline Age at diagnosis & 0.168 & 1 & 0.682 & 0.205 & 1 & 0.651 & 0.025 & 1 & 0.875 \\
\hline Tanner stage at diagnosis & 0.253 & 1 & 0.615 & 0.004 & 1 & 0.949 & 0.243 & 1 & 0.622 \\
\hline Treatment strategy & 0.626 & 1 & 0.429 & 0.248 & 1 & 0.248 & 0.758 & 1 & 0.384 \\
\hline Time & 7.621 & 3 & 0.055 & 21.199 & 3 & $<0.001$ & 23.855 & 3 & $<0.001$ \\
\hline Timextreatment strategy & 9.270 & 3 & 0.026 & 8.902 & 3 & 0.031 & 6.843 & 3 & 0.077 \\
\hline
\end{tabular}

GEE, generalized estimating equation; BMI, body mass index; DF, degree of freedom.

A

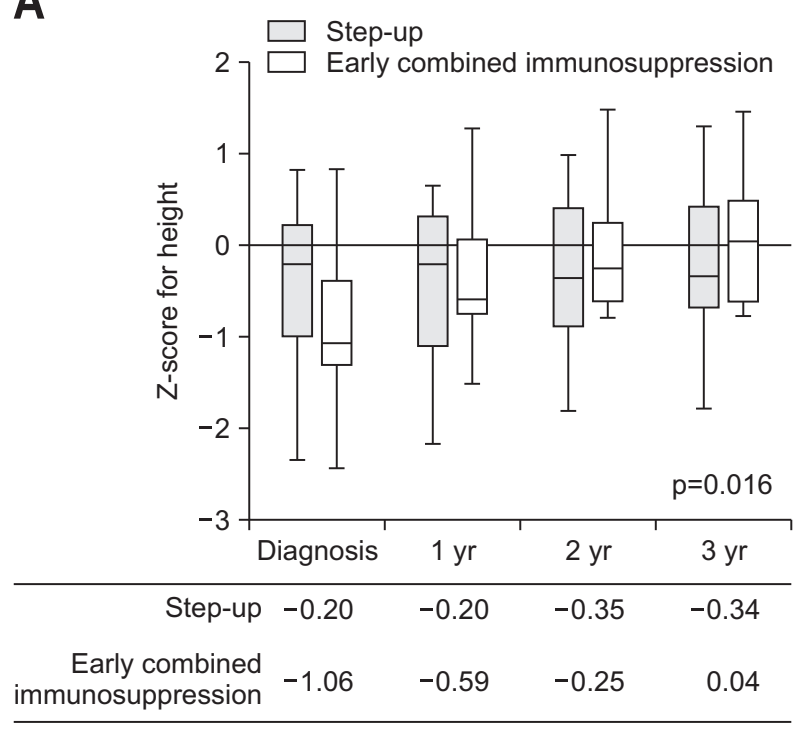

B

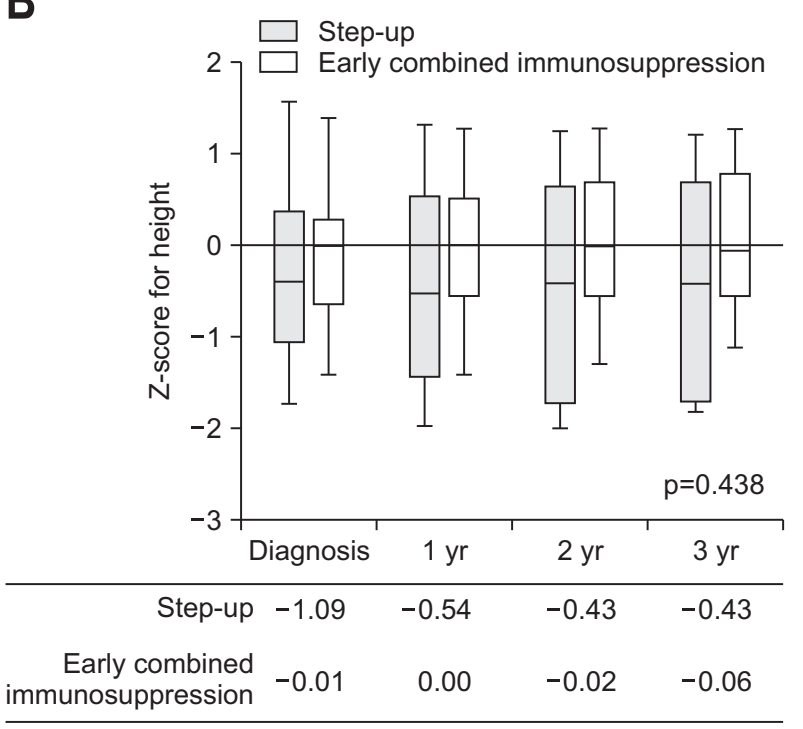

Fig. 2. Box-and-whisker plots of height $z$-scores since the time of diagnosis in subgroups further divided according to the Tanner stage. Box-andwhisker plot showing the median (line in box); 25th and 75th percentiles (box ends) and minimum and maximum (whiskers) values. Height zscores of patients in Tanner stages 1-2 each year after the time of diagnosis (A); and height z-scores of patients in Tanner stages 4-5 each year after diagnosis (B). The $\mathrm{p}$-values represent the interaction effects between the treatment strategies and time in the generalized estimating equation analysis. The table below represents the median height z-scores in each group in Tanner stages 1-2 and Tanner stages 4-5 shown in (A), and (B), respectively. 
The need for informed consent was waived by the board. And patient's records and information were anonymized and deidentified prior to analysis.

\section{RESULTS}

\section{Comparison of baseline characteristics between the two groups}

A total of 33 patients were included in this retrospective study. Sixteen patients had been allocated to the step-up group, and 17 patients to the early combined immunosuppression group. Median age at diagnosis was significantly lower in the step-up group when compared with the early combined immunosuppression group (12.1 years vs 15.0 years, $\mathrm{p}=0.009$ ) (Table 1). The duration from initial diagnosis to IFX infusion was also significantly longer in the step-up group compared with the early combined immunosuppression group (11.4 months vs 0.7 months, $\mathrm{p}<0.001$ ) (Table 1). As there was no patient identified as Tanner stage 3 at diagnosis, patients were classified as either Tanner stage 1-2 or 4-5 (Table 1).

\section{Comparison of z-scores for growth indicators between the two groups}

Although median height $\mathrm{z}$-scores from diagnosis showed gradual increase in the early combined immunosuppression group and stayed stable in step-up group, overall distribution of height z-scores failed to show apparent difference between stepup group and early combined immunosuppression group (Fig. 1A). In terms of weight and BMI, better improvements in the median $\mathrm{z}$-score are observed at 1 year after diagnosis in early combined immunosuppression group, but other figures show similar change between two therapeutic strategies (Fig. 1B and C).

GEE analysis enabled further consideration. The effect of treatment strategy on height was not significant when analyzed from diagnosis $(\mathrm{p}=0.626)$. The effect of time on height was also insignificant when analyzed from diagnosis $(p=0.055)$. However, the interaction effect between treatment strategy and time was significant in GEE analysis for $\mathrm{z}$-scores for height $(\mathrm{p}=0.026)$ and weight ( $\mathrm{p}=0.031$ ) starting from diagnosis after adjusting for sex, age at diagnosis, and Tanner stage at diagnosis, suggesting better improvement in z-scores for height and weight in the early combined immunosuppression group than the step-up group (Table 2). Meanwhile, when the z-scores for BMI were analyzed from diagnosis, GEE analysis failed to show a significant interaction effect between treatment strategy and time $(p=0.077)$ (Table 2).

\section{Analysis in subgroups further divided according to Tan- ner stage}

Height z-scores in subgroups of Tanner stage 1-2 revealed similar height patterns as in Fig. 1, with median height $\mathrm{z}$-scores nearly reaching $\mathrm{z}=0$ at 3 years after diagnosis in the early com-

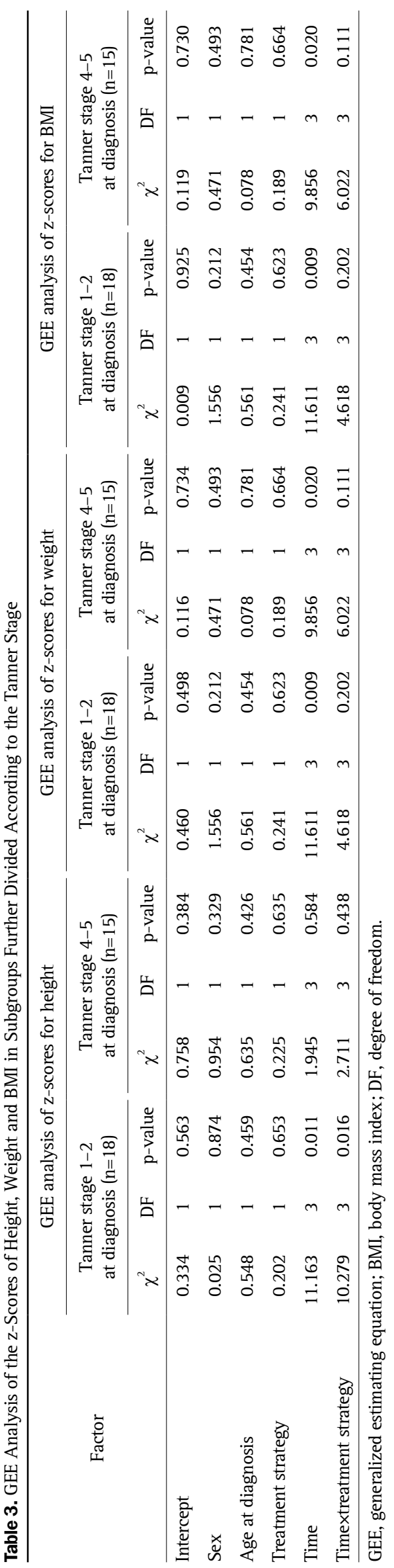


bined immunosuppression group (Fig. 2). GEE analysis of height z-scores among these patients revealed a significant time effect when analyzed from diagnosis, suggesting improvement of height z-scores overtime after diagnosis regardless of treatment strategies $(p=0.011)$. On the contrary, the effect of treatment strategy failed to prove significant results when analyzed from diagnosis, suggesting insignificant difference between treatment strategies in terms of height $z$-scores $(p=0.653)$. The interaction between the treatment strategy and time in z-scores for height when analyzed from diagnosis also proved significant after adjusting for sex and age at diagnosis, indicating better improvement in z-scores for height in the early combined immunosuppression group than the step-up group $(\mathrm{p}=0.016)$ (Table 3$)$.

In patients with Tanner stage 4-5, GEE analysis of height zscores failed to prove significant effect in terms of treatment strategy or time when analyzed from diagnosis. Furthermore, the interactions between treatment strategy and time were also insignificant when analyzed from diagnosis (Table 3).

The z-scores for weight or BMI from diagnosis showed similar trends of gradual increase in both treatment groups regardless of Tanner stage. GEE analysis was significant in terms of time regardless of Tanner stage, indicating significant improvement of weight or BMI overtime after diagnosis in both treatment strategies. On the other hand, GEE analysis failed to show significant result in treatment strategy or interactions between treatment strategy and time in terms of weight or BMI (Table 3).

\section{Adverse events}

No serious adverse event, such as infection or malignancy had occurred in the subjects. Minor adverse events of gastrointestinal disturbance $(n=4)$ and hair loss $(n=3)$ had occurred in six patients. However, no statistical significance was observed between the two groups $(\mathrm{p}=1.000)$.

\section{DISCUSSION}

This is the first study to compare the efficacy of early combined immunosuppression with step-up treatment strategy in terms of growth for a long-term period of 3 years after diagnosis in pediatric patients with moderate to severe luminal CD who had sustained clinical remission under combined immunosuppression with IFX and AZA. The long-term restoration of linear growth was superior in the early combined immunosuppression when height z-scores were analyzed for 3 years from diagnosis.

While a number of studies support the effect of IFX on growth, ${ }^{3,8,9,11,12}$ there is limited data regarding the effect of early combined immunosuppression on long-term growth restoration. Our study showed that an early combined immunosuppression strategy was superior to step-up strategy in improving longterm height z-scores starting from diagnosis after adjusting for sex, age at diagnosis, and Tanner stage at diagnosis. This significant difference between early combined immunosuppres- sion and step-up strategy on linear growth was also revealed in a subsequent subgroup analysis of patients with Tanner stage 1-2 after adjusting for sex and age at diagnosis, while no significance was revealed in patients with Tanner stage 4-5. These results indicate that the relative effectiveness of early combined immunosuppression on linear growth may only be expected when growth potential remains, suggesting the efficacy of early introduction of biologics on linear growth restoration in this particular group of pre-pubertal or early pubertal stages.

Growth failure has been recognized as a characteristic feature of pediatric patients with CD for more than 20 years. ${ }^{21,22}$ The degree of growth failure is prominent in patients who are diagnosed with CD in early pubertal stages (Tanner stage 1-2), and those who possess extensive intestinal inflammation at diagnosis. ${ }^{9}$ Moreover, up to $30 \%$ of patients continuously have linear growth retardation after treatment. ${ }^{23}$ Although the etiology of growth failure in $\mathrm{CD}$ remains unclear, several factors have been proposed to be interrelated with growth failure, while the two most important factors are chronic undernutrition and inflammatory cytokines secreted from the inflamed intestine. ${ }^{23-25}$ The long term use of $\mathrm{CS}$, one of the conventional mainstays in $\mathrm{CD}$, also increases the risk for growth failure. ${ }^{22}$

The 2014 ECCO/ESPGHN guideline recommends exclusive EN for at least 68 weeks instead of CS as the first choice for induction in pediatric CD patients. ${ }^{1}$ The primary induction with IFX is considered for patients with high risk factors including deep colonic ulceration on endoscopy, extensive (pan-enteric disease), marked growth retardation of $>-2.5$ height $z$-score, severe osteoporosis, stricturing and penetrating disease at onset, or severe perianal disease. ${ }^{1}$ While most previous cohorts have failed to reveal a restoration of height to a normal distribution, one recent study including luminal CD patients treated with IFX showed a restoration of height $\mathrm{z}$-scores to near normal levels of $\mathrm{z}=0$ when IFX was introduced earlier during the disease course. ${ }^{12}$ Although our study differs in factors such as disease severity at diagnosis, the criteria for patient inclusion, and group division, our findings correspond with the findings of Church et al., ${ }^{12}$ showing a restoration of height to a normal level of $\mathrm{z}=0$ in patients of the early combined immunosuppression group with Tanner stage 1-2. Combining the findings of our study with Church et al. ${ }^{12}$ and considering the unsatisfying compliance to exclusive EN that many physicians face in real-life clinical practice, we propose that on addition to initial exclusive EN treatment, biologics should be considered when continuous linear growth failure is noted in $\mathrm{CD}$ patients of Tanner stage 1-2.

The main role of AZA in combination therapy seems to be involved in the suppression of antibodies against IFX, which seems to benefit for 6 months to 1 year from treatment initiation. ${ }^{26}$ However, serious complications including infection or lymphoma such as hepatosplenic T-cell lymphoma (HSTCL) may complicate the use of AZA, especially in young males. ${ }^{27,28}$ These observations have led to a decrease in use of AZA among 
pediatric CD patients and methotrexate (MTX) has been widely used instead of AZA. However, there is scarce data regarding MTX usage in combination treatment. One randomized doubleblinded, placebo-controlled study failed to prove the additional benefit of combination therapy with IFX and MTX in comparison with IFX monotherapy. ${ }^{29}$ A recent review on the benefits and risks of combined immunosuppression therapy in pediatric IBD suggested that the usage of combination therapy may be indicated in patients having high risk of serious disease-related complications, while emphasizing the importance of a personalized strategy, aiming at balancing the risk and benefit, until more evidence accumulates in children. ${ }^{30}$

Our study possesses some limitations. First, the small number of subjects may not represent the actual patient population in real life practice. Second, the inclusion criteria may have posed a risk for selection bias. Long-term use of IFX and the sole inclusion of subjects who were under sustained clinical remission may have affected the analysis by selecting a subgroup of patients who responded well to treatment. Moreover, inclusion of only those who were under sustained clinical remission without consideration of endoscopic remission only partially eliminates the effect of inflammation on growth, as a discrepancy between clinical symptoms and endoscopic findings exists during IFX treatment. ${ }^{31}$ Third, the younger age at diagnosis in the stepup group may have contributed to a higher growth potential in the step-up group, possibly neutralizing the effect of early combined immunosuppression on linear growth. However, despite this significant difference in diagnosis age, Tanner stage did not show a significant difference between the two groups. This may have been due to the delay of puberty in pediatric $\mathrm{CD} .^{31}$ Moreover, despite the possible neutralizing effect due to the difference in age between groups, statistical significance was revealed between the two groups. Fourth, analysis regarding adverse events of early combined immunosuppression was limited in our study. Due to the intrinsic design of our study to include patients tolerable to long term use of IFX for 3 years, no major side effects were noticed during the observation period regarding combined immunosuppression. However, to date we have not experienced any life-threatening side effects including HSTCL or any malignancies. ${ }^{32}$ Further studies excluding the limitations in our study should allow more precise evaluation regarding the effect of early combined immunosuppression on linear growth.

In conclusion, although linear growth rate was similar between the early combined immunosuppression and the step-up group during the period of combination treatment, long-term restoration of linear growth was superior in the early combined immunosuppression when compared from diagnosis. This difference was also significant in patients in Tanner stage 1-2 at diagnosis. Early introduction of biologics at diagnosis should be considered in terms of improvement of linear growth in pediatric CD patients with Tanner stage 1-2.

\section{CONFLICTS OF INTEREST}

No potential conflict of interest relevant to this article was reported.

\section{REFERENCES}

1. Ruemmele FM, Veres G, Kolho KL, et al. Consensus guidelines of ECCO/ESPGHAN on the medical management of pediatric Crohn's disease. J Crohns Colitis 2014;8:1179-1207.

2. Caprilli R, Gassull MA, Escher JC, et al. European evidence based consensus on the diagnosis and management of Crohn's disease: special situations. Gut 2006;55 Suppl 1:i36-i58.

3. Hyams J, Crandall W, Kugathasan S, et al. Induction and maintenance infliximab therapy for the treatment of moderate-to-severe Crohn's disease in children. Gastroenterology 2007;132:863-873.

4. Hyams JS, Griffiths A, Markowitz J, et al. Safety and efficacy of adalimumab for moderate to severe Crohn's disease in children. Gastroenterology 2012;143:365-374.e2.

5. D’Haens G, Baert F, van Assche G, et al. Early combined immunosuppression or conventional management in patients with newly diagnosed Crohn's disease: an open randomized trial. Lancet 2008;371:660-667.

6. Khanna R, Bressler B, Levesque BG, et al. Early combined immunosuppression for the management of Crohn's disease (REACT): a cluster randomised controlled trial. Lancet 2015;386:1825-1834.

7. Lee YM, Kang B, Lee Y, Kim MJ, Choe YH. Infliximab “Top-Down” strategy is superior to "Step-Up" in maintaining long-term remission in the treatment of pediatric Crohn disease. J Pediatr Gastroenterol Nutr 2015;60:737-743.

8. Borrelli O, Bascietto C, Viola F, et al. Infliximab heals intestinal inflammatory lesions and restores growth in children with Crohn's disease. Dig Liver Dis 2004;36:342-347.

9. Mamula P, Telega GW, Markowitz JE, et al. Inflammatory bowel disease in children 5 years of age and younger. Am J Gastroenterol 2002;97:2005-2010.

10. Walters TD, Kim MO, Denson LA, et al. Increased effectiveness of early therapy with anti-tumor necrosis factor-alpha vs an immunomodulator in children with Crohn's disease. Gastroenterology 2014;146:383-391.

11. Crombé V, Salleron J, Savoye G, et al. Long-term outcome of treatment with infliximab in pediatric-onset Crohn's disease: a population-based study. Inflamm Bowel Dis 2011;17:2144-2152.

12. Church PC, Guan J, Walters TD, et al. Infliximab maintains durable response and facilitates catch-up growth in luminal pediatric Crohn's disease. Inflamm Bowel Dis 2014;20:1177-1186.

13. Hyams J, Walters TD, Crandall W, et al. Safety and efficacy of maintenance infliximab therapy for moderate-to-severe Crohn's disease in children: REACH open-label extension. Curr Med Res Opin 2011;27:651-662.

14. Cameron FL, Altowati MA, Rogers P, et al. Disease status and pubertal stage predict improved growth in antitumor necrosis factor 
therapy for pediatric inflammatory bowel disease. J Pediatr Gastroenterol Nutr 2017;64:47-55.

15. Levine A, Koletzko S, Turner D, et al. ESPGHAN revised Porto criteria for the diagnosis of inflammatory bowel disease in children and adolescents. J Pediatr Gastroenterol Nutr 2014;58:795-806.

16. Hyams J, Markowitz J, Otley A, et al. Evaluation of the pediatric Crohn disease activity index: a prospective multicenter experience. J Pediatr Gastroenterol Nutr 2005;41:416-421.

17. Levine A, Griffiths A, Markowitz J, et al. Pediatric modification of the Montreal classification for inflammatory bowel disease: the Paris classification. Inflamm Bowel Dis 2011;17:1314-1321.

18. Lee MN, Kang B, Choi SY, et al. Relationship between azathioprine dosage, 6-thioguanine nucleotide levels, and therapeutic response in pediatric patients with IBD treated with azathioprine. Inflamm Bowel Dis 2015;21:1054-1062.

19. Kang B, Choi SY, Kim HS, Kim K, Lee YM, Choe YH. Mucosal healing in paediatric patients with moderate-to-severe Luminal Crohn's disease under combined immunosuppression: escalation versus early treatment. J Crohns Colitis 2016;10:1279-1286.

20. Korea Center for Disease Control and Prevention; The Korean Pediatric Society; The Committee for the Development of Growth Standard for Korean Children and Adolescents. 2007 Korean Children and Adolescents Growth Standard (commentary for the development of 2007 growth chart) [Internet]. Seoul: Division of Chronic Disease Surveillance; c2007 [cited 2017 March 2]. Available from: http://cdc.go.kr/CDC/notice/ CdcKrInfo0201.jsp?menuIds=HOME001-MNU1154-MNU0005MNU1889\&cid $=1235$.

21. Griffiths AM, Nguyen P, Smith C, MacMillan JH, Sherman PM. Growth and clinical course of children with Crohn's disease. Gut 1993;34:939-943.

22. Motil KJ, Grand RJ, Davis-Kraft L, Ferlic LL, Smith EO. Growth failure in children with inflammatory bowel disease: a prospective study. Gastroenterology 1993;105:681-691.

23. Ballinger A. Fundamental mechanisms of growth failure in in- flammatory bowel disease. Horm Res 2002;58 Suppl 1:7-10.

24. Ballinger AB, Azooz 0, El-Haj T, Poole S, Farthing MJ. Growth failure occurs through a decrease in insulin-like growth factor 1 which is independent of undernutrition in a rat model of colitis. Gut 2000;46:694-700.

25. Kirschner BS, Klich JR, Kalman SS, deFavaro MV, Rosenberg IH. Reversal of growth retardation in Crohn's disease with therapy emphasizing oral nutritional restitution. Gastroenterology 1981;80:10-15.

26. Ungar B, Chowers Y, Yavzori M, et al. The temporal evolution of antidrug antibodies in patients with inflammatory bowel disease treated with infliximab. Gut 2014;63:1258-1264.

27. Cucchiara S, Escher JC, Hildebrand H, Amil-Dias J, Stronati L, Ruemmele FM. Pediatric inflammatory bowel diseases and the risk of lymphoma: should we revise our treatment strategies? J Pediatr Gastroenterol Nutr 2009;48:257-267.

28. Kotlyar DS, Osterman MT, Diamond RH, et al. A systematic review of factors that contribute to hepatosplenic T-cell lymphoma in patients with inflammatory bowel disease. Clin Gastroenterol Hepatol 2011;9:36-41.e1.

29. Feagan BG, McDonald JW, Panaccione R, et al. Methotrexate in combination with infliximab is no more effective than infliximab alone in patients with Crohn's disease. Gastroenterology 2014;146:681-688.e1.

30. Cozijnsen MA, Escher JC, Griffiths A, Turner D, de Ridder L. Benefits and risks of combining anti-tumor necrosis factor with immunomodulator therapy in pediatric inflammatory bowel disease. Inflamm Bowel Dis 2015;21:951-961.

31. Ballinger AB, Savage MO, Sanderson IR. Delayed puberty associated with inflammatory bowel disease. Pediatr Res 2003;53:205210.

32. Chun JY, Kang B, Lee YM, Lee SY, Kim MJ, Choe YH. Adverse events associated with azathioprine treatment in Korean pediatric inflammatory bowel disease patients. Pediatr Gastroenterol Hepatol Nutr 2013;16:171-177. 